\title{
¿Por qué hablar de bioética en la atención primaria en salud?*
}

\section{Why talk about bioethics in primary health care?}

\section{O porquê falar de bioética na atenção primária em saúde?}

\author{
Fecha de recepción: 12 de diciembre de 2015 \\ Fecha de evaluación: 21 de marzo de 2016 \\ Fecha de aceptación: 6 de abril de 2016
}

Disponible en línea: 11 de abril de 2016

Manuel Pérez Ayala*

DOI: http://dx.doi.org/10.18359/rlbi.1484

Cómo citar:

Pérez Ayala, M. A. (2016). ¿Por qué hablar de bioética en la Atención Primaria de Salud? Revista Latinoamericana de Bioética, 16(2), 102-117. DOI: http://dx.doi.org/10.18359/rlbi.1484

\footnotetext{
Artículo de reflexión.

** Psicólogo Clínico acreditado, de la Sociedad Chilena de Psicología Clínica; magíster en Bioética, de la Universidad de Chile. Docente de la Universidad Autónoma de Chile, sede Talca, región del Maule, Chile. Correo electrónico: mperezayala@gmail.com. Teléfono móvil: +56977590557 / +56966587604. ORCID: http://orcid.org/0000-0002-8450-6628. Talca, Chile.
} 


\title{
Resumen
}

Este trabajo tiene como objetivo plantear una reflexión sobre la necesidad de incluir de manera efectiva planteamientos bioéticos en la atención primaria de salud (aps) en el contexto latinoamericano, describiendo y proponiendo aspectos relevantes en los cuales la disciplina concretamente puede aportar.

Caracterizada la aps con prestaciones desplegadas, tanto a nivel individual, como comunitario, se describen sus desafíos futuros y el horizonte mismo que la bioética tiene en ella, con lo cuals e contribuye a optimizar el cuidado de la salud y así se dota de excelencia el actuar profesional y sus procedimientos, y se humaniza el acto de atención en salud pública.

Se concluye que los criterios que abogaban por la cantidad en este nivel de salud afectaron negativamente a la expresión de una calidad total hacia los beneficiarios; también ha mermado el impacto de la bioética asimilada en la salud pública. Superada esta transición, la bioética es llamada a integrar la calidad en salud, lo que a su vez acorta significativamente la brecha existente hacia la equidad.

Palabras clave: Bioética, atención primaria de salud, salud pública, calidad, equidad.

\begin{abstract}
This work aims to propose a reflection on the need to include bioethical approaches effectively in Primary Health Care (PHC) in the Latin American context, describing and proposing relevant aspects in the particular discipline can contribute.

Characterized PHC services deployed with both individual and community level, described its future challenges and the same horizon that bioethics has in it, help optimize the health care professional providing excellent acting and its procedures, humanizing the act of public health care itself.

It is concluded that the criteria advocated quantity at this level of health adversely affected the expression of total quality to beneficiaries, the same that has reduced the impact of bioethics assimilated on public health. Once this transition, bioethics is called to integrate health quality, which in turn significantly shortens the gap towards equity.
\end{abstract}

Keywords: Bioethics, primary health care, public health, quality, equity.

\section{Resumo}

Este trabalho tem como objetivo levantar uma reflexão sobre a necessidade de incluir de maneira efetiva abordagens bioéticas na atenção primária à saúde (APS) no contexto latino-americano, descrevendo e propondo aspectos relevantes nos quais a disciplina pode dar uma contribuição concreta.

A APS caracterizada com serviços espalhados, tanto em nível individual, como comunitário, descrevem-se seus desafios futuros e do próprio horizonte que a bioética tem em ela, com o que contribui para otimizar os cuidados de saúde e, assim, dota-se de excelência o agir profissional e seus procedimentos, e é humanizado o ato de cuidados de saúde pública.

Concluiu-se que os critérios que preconizavam pela quantidade neste nível de saúde afetaram negativamente à expressão de uma qualidade total para os beneficiários; também tem diminuído o impacto da bioética assimilada na saúde pública. Uma vez que esta transição tem sido superada, a bioética é chamada para integrar a qualidade da saúde, que por sua vez reduz significativamente o fosso que existe para a equidade.

Palavras-chave: Bioética, cuidados primários de saúde, saúde pública, qualidade, equidade. 


\section{Introducción}

En la Asamblea General realizada en 1977, la Organización Mundial de la Salud (OMS) plantea como imperiosa la necesidad de garantizar el acceso a la salud para la mayoría de las personas - la ambiciosa meta propuesta era "a todos"-, proyectando la fecha para su complimiento el año 2000. Al año siguiente, en la reunión llevada a cabo en Alma Ata (Kazajistán), se estableció que para alcanzar el objetivo propuesto se necesitaba una estrategia diferente a las desplegadas hasta entonces, a la que se denominó atención primaria de la salud (APS).

La Declaración de Alma Ata (oms, 1978) explicita el acento en el desarrollo y fortalecimiento de los establecimientos de salud con el imperativo ético de la equidad, a través de mejorar el acceso a la salud. En ella se define a la APS como "el cuidado esencial de la salud en base a métodos científicos y socialmente aceptables y a tecnologías universalmente accesibles a toda persona y familia de la comunidad a través de una participación plena y de unos costos que estén al alcance de la cada país y de la comunidad" (oms, 1978, pp. 1 y 2). Se deja así establecido que es el primer nivel de contacto de cada individuo con el sistema sanitario, que lleva los servicios de salud lo más cerca que sea posible de los lugares donde viven y trabajan las personas, y se instaura como el primer elemento del proceso constante del cuidado de la salud.
Más allá de los determinantes sociales de cada región y país, la estrategia propuesta es aceptada e implementada en función de la desigualdad existente en la condición de la salud de las personas, especialmente entre los países denominados en vías de desarrollo y desarrollados, e incluso también de la brecha a este respecto existente dentro de cada nación.

Luego de la Declaración de Alma Ata, los diferentes tratados e informes internacionales que le sucedieron (Organización Panamericana de la Salud [ops], 2007 y 2008, por nombrar dos documentos latinoamericanos) brindan un marco normativo para que se coordine y articule el diseño de la atención integral, velando ante todo por los derechos básicos concernientes a la salud. La perspectiva que otorga esta adjudicación de derechos, aplicados en salud, se refiere a que es un componente inalienable de la dignidad humana y se trata de un bien en sí mismo, y que por tanto no requiere una mayor justificación. Autores como Ase y Burijovich (2009) explican que, dado lo anterior, todas las personas, por el hecho de existir, tienen derecho a la salud; Outomuro (2013a) refina señalando que de manera errónea se ha hablado de "derecho a la salud", ya que este es imposible de satisfacer a cabalidad, no así el derecho a recibir asistencia sanitaria, que sería lo correcto de manifestar y exigir.

Concediendo entonces el hablar sobre el derecho a la atención sanitaria, la cartera de servicios que propone la 
APS — concebida ya como la puerta de entrada a la red de salud, mediatizada por el cuidado continuo- reúne las competencias necesarias para la aplicación de políticas públicas que apunten a satisfacer ese derecho, así como a mejorar las condiciones generales de salud de la población.

\section{Características de la atención primaria de salud}

En el nivel primario de atención en salud, en el cual se han privilegiado las intervenciones cercanas de tipo familiar con un enfoque comunitario y de carácter territorial, no se trabaja - o al menos no se debieran proyectar así los abordajes - considerando tanto el corto, cuanto el medio o el largo plazo. Su objetivo, por lo demás, no es la intervención o el tratamiento de los problemas concretos de salud, sino el manejo global de los usuarios y usuarias que allí concurren por equipos de cabecera; a los beneficiarios en todo su ciclo vital no se les ve como sujetos aislados, desligados de su medio natural, de sus contextos sociales y humanos, sino como integrados en sus estructuras familiares y comunitarias (Gracia, 2001). En la APS el pilar es el equipo de salud centrado en mejorar la accesibilidad de prestaciones de salud a los usuarios/usuarias y sus familias (Zurro et al., 2014) y se caracteriza por ser globalizadora, integrativa y de duración longitudinal, hablando sobre las atenciones entregadas. Características centrales son asimismo el énfasis promocional y preventivo —-más que curativo- de sus estrategias, que planifican actividades más educadoras que restauradoras.

En general, estas estrategias utilizadas para el logro de sus objetivos son el desarrollo de mecanismos de gestión participativa — dirigidos principalmente hacia los trabajadores y la comunidad en general_-, la consolidación de equipos de salud multidisciplinarios con fuerte vocación comunitaria y el fomento de la responsabilización de estos mismos equipos sobre la población por atender. La APS es considerada como una forma de organización del sistema de salud tendiente hacia el logro de la equidad en el acceso, la programación local de los servicios y, en la medida de lo posible, la democratización del sistema de salud y la sociedad (Yavich et al., 2010); todo a través del activo estímulo de la colaboración comunitaria.

Al respecto, es reconocible que la participación de la comunidad en los dispositivos de salud esté mediatizada por la fuerza de una necesidad a veces costosa, la que se esperaría que fuera reemplazada a través de un plano de justicia (Rueda, 2013), que exija el cumplimiento de una ética de mínimos aceptables (Cortina, 2000; Escríbar, 2004) para aspirar a un estado de salud cabal de las personas. Como en Latinoamérica un importante número de población que es beneficiaria de la APs habita en espacio social rural, se deben tener presentes las particulares relaciones que tienen lugar en tal área y territorio (Romero, 2012), cuyos centros referenciales de acogida en salud más 
próximos van desde postas de salud rural, consultorios, centros comunitarios y centros de salud familiar, hasta hospitales de baja complejidad —en algunos países hoy llamados hospitales comunitarios_- donde se da lugar a la manifestación natural de sus inquietudes, necesidades y requerimientos, debido a la interacción diaria de atención que en ellos converge.

En este escenario particular de accionar se concibe que el sistema de atención en salud cumpla al menos con tres aspectos cruciales: accesibilidad, eficiencia y adaptabilidad (Outomuro, 2013). Que sea accesible se refiere a lo geográfico, económico, cultural y legal. Lo primero no solo se mide en distancia, sino sobre todo en tiempo; lo segundo guarda relación con la forma de pago de los distintos servicios recibidos e intrínsecamente está supeditado al poder adquisitivo de la población y de la macro-meso política sanitaria implementada en cuanto a quién (y cómo) costea dichos gastos. El factor cultural no es menor a la hora de recibir atención en APS, como se abordará en la discusión respecto a los desafíos venideros, debido al perfil del grueso de su población beneficiaria, ya que si bien puede existir gratuidad en buena parte de los servicios prestados, las llamadas barreras culturales pueden significar un impedimento para su correcto y oportuno acceso. Por último, desde el área legal se pueden desprender algunos obstáculos vinculados a las distintas formas de atención, específicamente al tipo de prestaciones, considerando usuarios y usuarias que por variadas razones no cuenten con cobertura para recibirlas.

Continúa Outomuro (2013) planteando que la eficiencia — capacidad de producir cierta cantidad de servicios en relación con el esfuerzo realizado- se entiende como la maximización de la cantidad del producto o la minimización del insumo; aquí el insumo corresponde al gasto económico, cantidad de personal requerido, tiempo empleado, entre otros, para la ejecución de las atenciones. Finalmente, la adaptabilidad refiere el carácter dinámico de la estructura desde donde se llevan a cabo las mencionadas atenciones, que caracterizadas por lo transversal de su inclusión, debe poseer la flexibilidad suficiente para permitir la adecuación a los cambios, tanto esperables como no esperables, que la diversidad poblacional presente.

\section{¿Por qué hablar de bioética en la atención primaria de la salud?}

Según la oms (1978), esta mencionada transversalidad de la APS se apoya en la construcción de consensos interinstitucionales levantados desde cada organización y no en la imposición u orientación sobre fórmulas fijas y repetitivas de políticas provenientes de una jerarquía vertical, ya que la participación de distintas organizaciones en la formulación de propuestas consensuadas le otorga mayor legitimidad a los procesos de política pública y mejora sus cada vez más altos y exigentes estándares: la 
puerta de entrada a la red de salud está radicada en el nivel primario, y es por esta condición de primera acogida en la red que los dilemas o conflictos que se generan a nivel de la APS repercuten directamente en los niveles superiores (León et al., 2010). Lo anterior puede generar pérdida de confianza en las organizaciones y finalmente redundar en una relación de menos calidad, con todos los efectos inmediatos y posteriores que esto pueda acarrear.

En las organizaciones de salud, ante todo durante la última década, se ha estimulado y así prosperado un creciente y general interés sobre la importancia del accionar ético de las personas que ahí laboran (Bedregal, 2012), dirigidas hacia el fomento de la excelencia -sinónimo de calidad- de sus profesionales; para que un individuo ejerza determinado oficio es necesario que él/ella sea primero un buen (excelente) profesional y, después, un profesional bueno (Gracia, 2000), y es que cada vez más se aspira a lograr una alta calidad ética institucional. Esta calidad ética se basa, primero, en impulsar un ambiente en el que prime el profesionalismo, en la gestión orientada a la calidad del servicio y su calidad ética, y en el diálogo con el contexto y especificación de su marco de responsabilidad social. En este entramado el profesional de salud tiene que aprender el correcto procedimiento de su práctica no solo de los hechos clínicos, sino también de los valores y los deberes (Gracia, 2012), incidiendo, directamente, la bioética en la asimila- ción formativa como en la ejecución de ese aprendizaje. Ahí resalta el contenido disciplinar de la bioética, por ejemplo, en la suposición prioritaria por parte del equipo de salud al evaluar y proyectar intervenciones en el beneficiario/a y en sus familiares, considerándolos como parte activa y no pasiva de ese equipo de trabajo (Rico y Domínguez, 2012). Abandonar una beneficencia paternalista por una beneficencia que promueva la autonomía es una directriz que expresa los principios comprendidos en la ética profesional, reflejada en un trato consciente de cada contexto, cuidadoso y de excelencia.

En el marco de la APS confluyen una serie de cuestiones éticas que han recibido escasa atención porque en determinadas ocasiones no son percibidas como tales, y que requieren una observación meticulosa y rigurosa. Algunas de las características éticas diferenciales que son propias y excluyentes de la atención primaria (Did Núñez, 2000) son la continuidad en la atención a lo largo del tiempo, lo que implica un mejor conocimiento del usuario/a como persona, incluyendo sus preferencias, sistema de valores, capacidad de autocuidado, etc., la perspectiva global, teniendo en cuenta sus circunstancias familiares y socioculturales, que cumple un rol de colaboración o de dificultad para la aplicación de medidas que diferencien y promuevan indistintamente el funcionamiento autónomo; y, como síntesis, la atención integral, no solo asistencial, sino con énfasis preventivo 
y de promoción de salud, que es conformada por actividades de educación que suponen directa conexión con las necesidades y valores de la población, lo que denota la proximidad y nivel de conocimiento sobre esta.

Al hablar directamente de indicadores de carácter bioético presentes en el APS, estos están sustentados, por ejemplo, en lo extensivo y vinculante de la responsabilidad profesional y en una amplia gama de actividades que van desde el mínimo que representa el cumplimiento de una cita acordada hasta la atención con resultados eficientes y el cuidadoso apego a cada uno de los beneficiarios. Este hecho se correlaciona con la responsabilidad de los usuarios/as y de sus familiares en el cumplimiento de los deberes de autocuidado, en el vasto campo de la prevención, el fomento de la salud y en la adherencia al tratamiento a veces esquiva, refieren Rico y Domínguez (2012), quienes a su vez destacan ya como hitos bioéticos más reconocidos la dignidad apreciada tanto para recibir una atención clínica común, como en la realización de un procedimiento puntual; el trato digno en la atención personalizada, cuidadosa de las características de las personas en cuestión, evitando — previa identificación-caer en facilismos paternalistas tan propios de este nivel (Couceiro, 2012), procurando la entrega completa de información y no centrándose solo en etapas que puedan interesar más al profesional, como el tratamiento, dejando de lado la exploración de las necesidades de los consultantes y la respuesta clara y pertinente a sus problemas y dudas personales.

Aspectos como los mencionados dan cuenta de que hablar de bioética en APS es aportar con un marco regulatorio marco ético- que ayude a desarrollar la complementariedad del equipo de salud, a refinar el diseño de intervenciones y a reflexionar sobre las estrategias y procesos que en un afán beneficioso, resolutivo y promovedor de autonomía, integre a los interesados en un papel activo dentro de los procedimientos ahí establecidos como parte de un actuar virtuoso y ético en el marco general de la salud pública. En sí, hablar de los atributos que la bioética puede aportar a la APS no es otra cosa que apuntar a la calidad.

\section{Agenda actual de la bioética en la atención primaria de la salud: calidad y equidad}

Aunque en el campo de la ética de la salud pública destacan áreas como preocupación por derechos individuales y bien común, vigilancia, regulación y justicia social en el marco de la salud global (Thompson et al., 2003), esta es distinta de la bioética debido a las amplias definiciones, implicaciones y alcances de la salud pública. Bioética y ética de salud pública presentan nociones e intereses comunes que se complementan de manera recíproca, como en la comprensión del campo teórico desde el cual ha emergido la ética en salud y las causas/efectos de las inequidades 
en este plano; ambas, a distintos niveles, son necesarias de incorporar en la concepción de la entrega de salud como en cada prestación y servicio otorgado.

Solo desde hace menos de dos décadas los teóricos e investigadores de la bioética comenzaron a interesarse explícitamente por la salud pública, ya que es conocido que desde su inicio la bioética se desarrolló en el campo biomédico y en áreas hospitalarias (Callahan y Jennings, 2002), donde la atención de primer contacto y los procesos de la salud pública fueron relegados a segundo término, a pesar de su trascendencia en todo sistema de salud. Ya consolidada como disciplina ampliada de la ética estrictamente médica — trasladada más allá de la relación clínica (Gracia, 2002)_, es necesario que elementos de calidad total y los aspectos principales de la bioética se enfoquen en el primer nivel de atención a la salud, en el cual son de mayor relevancia los fenómenos antropológicos, sociológicos y político-comunitarios, centrados en una relación con determinadas particularidades y una moderna concepción de la salud pública (Domínguez Márquez y Manrique Nava, 2011), que son fiel reflejo de la equidad o la ausencia de esta.

Bioética y equidad han sido relacionadas y estudiadas por variados autores en el contexto latinoamericano (Lolas, 2001; Ferrer, 2003; Garrafa y Porto, 2003; León, 2010b), que concuerdan en la necesidad de imprimirle un sello particular en nuestro territorio. Con barreras por franquear aún, de los pri- marios propósitos social y educacional se incluye luego a la salud, en los últimos años con la medida de la calidad. Hablar puntualmente de calidad y equidad en la salud pública contiene la complejidad de ser conceptos multidimensionales y polimodales. Mientras la calidad en salud, si bien agrega requerimientos y nuevos ítems a las exigencias que le son propias, atiende básicamente a parámetros de buenas prácticas y búsqueda de excelencia definida e identificable desde el momento de la formación profesional hasta el producto final que sería la prestación en sí. La significación de excelencia representa una calidad técnica resultante del entrenamiento intelectual que transforman a su autor/ autora en una persona virtuosa, plantea Drumond (2010). Un profesional asegura una atención de calidad luego de desarrollar ciertas habilidades cognitivas y emplearlas realizando algo útil para la sociedad. No basta saber una técnica ni perfeccionarla, es necesario que su finalidad atienda a una demanda social; se precisa que conlleven el contexto de la sociedad, que sean útiles y que promuevan satisfacción para quienes sirven (Drumond, 2010), con el requerimiento de alta exigencia que la actividad de la salud plantea en la actualidad.

Por su parte, la equidad — concebida esta como el establecimiento de reglas justas para la sociedad o respeto a la adecuación de las normas genéricas para cada contexto específico (Drumond, 2010)_ en salud pública puede significar la opción de tratar desigualmente a 
los desiguales, ya que el acceso a bienes y servicios en países de bajo desarrollo social, como los existentes en América Latina, contiene un carácter punitivo para las ciertas poblaciones de centros urbanos o en el medio rural, como es el caso del perfil medio de consultantes en APs. Los dilemas asociados, por ejemplo, a los modos de distribución o limitación de recursos escasos en materia sanitaria poseen respuestas distintas, sea que se dé mayor o menor importancia a los criterios de equidad (Zúñiga, 2008) y de garantía de cuidados integrales y necesarios para una vida saludable, que presupone prevención, promoción y tratamientos entregados por profesionales competentemente formados.

Representan estas acciones preventivas y promocionales aspectos simples pero contundentes de la doctrina priorizada en APS, componentes estructurales básicos de la atención a este nivel (Outomuro, 2013b), como una metodología para abordar la causalidad de los diferentes padecimientos en el núcleo de la familia y en su dinámica psicosocial. Sin embargo, los esquemas de atención familiar han tropezado, entre varios, con la hasta ahora infranqueable traba de contar con recurso humano limitado y atender grandes cantidades de usuarios/as por jornada, lo que atenta y en muchos casos francamente impide la calidad (Domínguez Márquez y Manrique Nava, 2011). La intervención planificada que no incorpora en su esquema una participación global y transversal de atención de y en familias ante eventos clínicos, consejerías individuales o familiares, estudios de familia, visitas domiciliarias integrales, talleres y charlas sobre temáticas obtenidas desde la comunidad misma, por nombrar las significativas, finalmente no puede llegar a la calidad propuesta y esperada.

Cada persona que conforma una sociedad determinada, concebida esta como comunidad civil e informada sobre las utilidades, riesgos y criterios éticos en juego, tiene el derecho y el deber de participar en regular su propia salud (Ortiz y Palavecino, 2005). La bioética puede encauzar la búsqueda de igualdad en las condiciones de vida deseadas, dotando de calidad a las atenciones y con el logro de la equidad en sus necesidades (Rozo, 2011), ya que por su carácter pluralista una de sus misiones es crear espacios de reflexión para que los involucrados argumenten, disientan y consensuen de manera práctica acuerdos comunes que les beneficien no solo en el proceso salud-enfermedad, sino también en la salud como estilo de vida, cultura, entretenimiento, deporte o como relaciones adaptativas con el medio ambiente. Los usuarios/as merecen una atención de calidad y excelencia, y estos son valores que deben incorporar los equipos y profesionales de la salud (León, 2008), a través de una estrategia de atención más cercana, de comunicación efectiva. Abordable desde una relación personal y humanizada, edificada en la confianza que entrega la proximidad del contacto continuo, ya que existe el deber ético profesional de promover la competencia 
en quienes atienden, procurando prescindir o al menos reducir al mínimo la intervención paternalista, gestionando una libertad informada y responsable.

La agenda actual de la APs, en la cual la bioética contribuye a desarrollar la calidad en términos de respuestas anticipatorias a las necesidades de la población, podría representarse en dos esferas. Primero, en el ámbito particular de la atención en salud, donde reside el propósito de conseguir una mejora continua, ya que entre los reclamos más frecuentes de la población se encuentra la llamada "deshumanización" que progresivamente ha entrado al box de atención clásico, es decir, cuando se otorga atención con un "alejamiento" o desconsideración tal que pudiera lesionar de manera alguna la dignidad de las personas (Domínguez Márquez y Manrique Nava, 2011) y ser así maleficente. Al tratar sobre calidad en APS es conveniente realzar el papel de los elementos bioéticos que suelen pasar desapercibidos mediante sus componentes de valores y principios éticos, o su mención es sumamente abstracta en cuanto a dificultar su cabal comprensión. No puede haber calidad total si no hay dignidad en la atención — escaso tiempo o postergación consecutiva de esta-, falta de responsabilidad o subrogación constante, o anulación del ya discutible concepto de autonomía.

Segundo, desde una panorámica general — servicios, programas nacionales de salud; sistema sanitario en sí- en la que se comprendan los valores fundamentales que afectan desde los bien- estares individuales hasta la estabilidad social, pertinentemente reconocidos e interiorizados previo a la elaboración de directrices. La atención en salud requiere incorporar una mirada bioética amplia, crítica y permanente que delibere y, de manera eventual, ayude a rediseñar su quehacer constante o al menos con celeridad cada vez que se aprecie como necesario. Kottow (2012) destaca además que en el entramado de la atención en salud hoy es posible detectar como grandes áreas de reflexión a las acciones específicas de salud pública y al debate sobre justicia social y global, tan aplazada como necesaria en América Latina. Siendo la APs central en el sistema sanitario, robustecer la política de salud pública también debiera ser una prioridad (Bass, 2015), debido a la influencia y reciprocidad entre salud y bienestar de una población (Calonge y Rodríguez, 1998), planteada así la equidad como parte fundamental del desarrollo político, social y económico de cada país, y objetivo central a la vez.

\section{Desafíos para la atención primaria de salud y horizonte próximo de la bioética}

Existen países señalados como prósperos que llevan décadas trabajando y perfeccionando sistemas de salud de cobertura universal, cuyas economías hacen que sus ciudadanos exijan cada vez más y mejor atención en salud. Distinto es el caso de los países en vías de desarrollo (Couceiro, 2008), que si bien han asumido como una obligación moral hacer 
efectivo el derecho a la asistencia sanitaria de sus ciudadanos, se encuentran inmersos en un marco sociopolítico y económico que en gran medida lo dificulta; por esto puede parecer lejano el hablar de calidad —y de bioética como un vehículo para esta_ cuando existen situaciones en las que aún se lucha por el derecho a la asistencia sanitaria, al acceso equitativo de cada ciudadano a estos servicios y, cuando se accede a estos servicios, las coberturas otorgadas sean deficitarias.

A pesar de los acuerdos firmados y los compromisos mediados, en la mayoría de los países del continente latinoamericano es difícil vislumbrar una pronta superación a las desigualdades en salud (Cardozo y Mrad, 2013); no se han establecido acciones verdaderas para solucionar los más graves problemas, por el incentivo predominante del trabajo hacia la competitividad, la innovación, el desarrollo tecnológico e impulso empresarial, sobre la accesibilidad en condiciones de miseria, pobreza o aislamiento, la solidaridad en su conjunto y el respeto estricto a las reformas de salud que hacia la calidad debieran apuntar.

Se debe trabajar para que en la APS se reconozcan y validen sin excepción principios como el de la justicia social, e integren en su proceder premisas éticas y bioéticas, para generar así un espacio de respeto profesional y moral para los usuarios/as, su comunidad y la sociedad por extensión (Did Núñez, 2000), y con esto favorecer la capacidad de comprender la información y decidir de manera libre y exenta de coerción alguna. Esta toma de decisiones, más las distintas versiones que pueda tener la entrega de calidad total y los procesos que puede guiar y ayudar a estructurar la bioética, conforma una tríada que es necesario analizar en su trascendencia social, laboral y consecuencial, según Rico y Domínguez (2012). Para lograr un óptimo trabajo en salud a través de la integralidad de las atenciones ofrecidas en las que se encamine la mejora de los estilos de vida de la población (Oyuela, 2010), se debe apuntar también a superar las problemáticas propias de este nivel, como son el progresivo aumento de cobertura, stock básico de medicamentos limitado, la restringida cobertura de patologías, el tiempo de espera de las derivaciones, entre otros.

Demarcando estas denominadas problemáticas, dentro de los desafíos más complejos venideros en la APS, destacan al menos tres, de distinta índole y cercanía de superación en el tiempo: 1. disminuir la brecha del recurso humano existente y pensar en el cómo de la redistribución del número total de atenciones y tipo de prestaciones; 2 . aumentar el acceso a especialistas de referencia —entendida su escasez- para reducir las listas de espera; y 3. la incorporación de un enfoque multicultural hablando no del nivel macro, sino de la consideración de cada región y su modos vivenciales, procurando el respeto, preservación y mantención de la identidad local en las comunidades que es relevante a la hora de planificar e implementar estrategias de 
atención diseñadas desde el alto nivel, y su adaptación a cada realidad contextual que, previo trabajo coordinado, en sí determinará la aplicación soci-culturalmente adecuada del relato bioético.

Independiente aún de esta anhelada adaptación del modelo a cada contexto sociocultural, la APS se encuentra en una etapa de cambio que busca, manteniendo el nivel técnico de sus profesionales, ampliar y en ciertos casos modificar las estrategias y disposiciones tanto individuales, como de equipo con las que se relacionen ante los usuarios, desde el ya conocido modelo biomédico clásico centrado en la enfermedad, a uno más integrativo de todas las cualidades de los individuos y sus familias, conocido como modelo biopsicosocial (León et al., 2010). En este modelo, desde la perspectiva y criterios de la bioética, proyectar estudios y luego abrir líneas investigativas sería de un inmenso aporte para identificar puntos críticos en los cuales intervenir, lo que aún se ve como una tarea distante. En el horizonte cercano se puede hacer una contribución imprescindible para mejorar la calidad de la salud y humanizar el acto mismo de atención, a partir del análisis de las complejidades propias de la práctica diaria de cualquiera de los profesionales, técnicos y funcionarios que trabajan en tales centros de salud. En específico, los alcances de la bioética en APS sería dirigir su actuar en lo individual y en lo comunitario, transitando de un encuentro puramente clínico a uno que traspase las paredes del box clásico, y promover la equidad social en cuanto al acceso, trato, forma y tipo de atención recibida, operando de tal forma en concordancia con las necesidades sanitarias de gran parte de los países de la región latinoamericana (Parenti, 2002). Junto a esto, se debe incentivar la autovigilancia en salud, así como guiar para formar una ciudadanía vigilante y crítica a través de educación para la salud, que debería ser el eje de todas las acciones del primer nivel de atención: la salud para las personas inicia desde la deliberación de los propios involucrados.

En la misma línea, la bioética puede encontrar una vía de ingreso para validarse e institucionalizarse en la Atención Primaria de Salud a través del Comité de Ética Asistencial (CEA) (León et al., 2010), una entidad básica en la consideración actual para el logro de calidad. El desarrollo y regular funcionamiento de un CEA plantea una inestimable circunstancia para estudiar y ayudar a resolver aquellos casos tanto de perfil clínico, como comunitario que en su evolución puedan contener conflictos o dilemas éticos, así como de generar protocolos de acción tendientes a estandarizar el enfrentamiento de esas problemáticas frecuentes en este nivel de atención.

Esta propuesta además es interesante y acertada (León et al., 2010), dada la posibilidad de iniciar con él un proceso de docencia centrada en la actualización de temas pertinentes a los miembros del comité y en la difusión de los conceptos generales de la bioética en la práctica profesional diaria de todos los miem- 
bros del equipo de salud, a través de cursos formativos, jornadas o talleres de reflexión y charlas abiertas a la comunidad. En general, permitiría una capacitación continua que se haría extensiva más allá de los mismos funcionarios y funcionarias de la salud, promoviendo la participación ciudadana activa, con lo cual también se aporte a disminuir la brecha hacia la equidad.

\section{Conclusiones}

La APS comprende un concepto interactivo de entender la salud que no funcionaría, o al menos no rendiría los frutos esperados, si no se obtuviera la retroalimentación directa y continua de los usuarios/as, es decir, información levantada y validada solo de primera fuente, que identifique y promueva correctas prácticas y evite las incorrectas; paternalismo como ejemplo latente de las últimas. La transversalidad de la APS llama a integrar distintas miradas que complementen la estrictamente médica y que por lo mismo expanda su rango de acción al equipo de salud, en tanto que las intervenciones se planifiquen comprendiendo la colaboración activa de la población a través de estrategias con abordajes interdisciplinarios, integrando prudentemente cada multiculturalidad.

Han pasado más de tres décadas desde la Declaración de Alma Ata y hace ya más de una década que formalmente se habla de bioética en APS: ipor qué los contenidos de esta disciplina no se han implementado aún?; en el caso que se hayan implementado, isu impacto ha sido significativo? Una respuesta breve es que aún estamos entrampados en una visión paternalista que fomenta un mal entendido asistencialismo, en el cual el criterio de la eficacia y maximización de recursos anacrónicamente se prorroga basado de forma excluyente en la evaluación de la cantidad. Entendible era que cualquier nueva estrategia debiera pasar antes por este tamiz de coste/ cuantía, lo que finalmente funcionaba como una matriz castradora que coartaba la creatividad de abordajes que - aunque innovadores y viablesno se implementaran a la luz de esos parámetros; inconcebible es que aún se siga haciendo.

Oportuno e inexcusable se hace incorporar en el presente la concepción de eficiencia que promueve la calidad con base en las necesidades de los consultantes, por los resultados inequitativos o discriminatorios que pudieran llegar a producir (Zúñiga, 2008) en su falta o equívoca aplicación. Compete a la bioética —mediante quienes le estudiamos e intentamos llevarla a la práctica-impulsar el diálogo ciudadano y exhortar la participación a través de un debate sociocomunitario, estimular el trato humanizado y atención de excelencia a través de medidas acordadas, inducir a la deliberación del profesional e impedir la intervención sin reflexión con el componente de crítica que hace que cada actor en esta trama de prestaciones asuma un rol activo y protagónico en el debate de la salud, la atención sanitaria y 
las ideas sobre la búsqueda de bienestar que motiva ese encuentro interpersonal, comunitario y social.

¿Se debe seguir hablando de bioética en APs? Identificando y superando el contraste entre los intereses de los usuarios/ as y quienes trabajan en y para la salud, el aporte de la postergada bioética a la atención de calidad puede también acortar significativamente la brecha aún existente hacia la equidad, teniendo en cuenta como objetivo general el fortalecer y modernizar el sistema de atención pública de salud. Se debe entonces continuar dialogando desde la bioética en APS hasta que su incorporación se vea reflejada tanto en la planificación, como en la ejecución de las prestaciones a este nivel, y de ser una necesidad se utilice como una conocida herramienta de oportuna y efectiva respuesta a un determinado requerimiento. La bioética, como parte y parámetro de excelencia, mediante la calidad, proporciona un atajo hacia la equidad.

\section{Referencias}

Ase I. y Burijovich J. (2009). La estrategia de atención primaria de la salud: iprogresividad o regresividad en el derecho a la salud? Salud Colectiva; 5(1), 27-47.

Bass, C. (2015). Propuesta para una política de desarrollo profesional continuo del recurso Médico de la Atención Primaria de Chile. Revista Chilena de Salud Pública, 19(3), 284-292.

Bedregal, P. (2012). Ética en las organizaciones de salud. En J. P. Beca y C. Astete
(Eds.), Bioética clínica (pp. 513-524). Santiago de Chile: Mediterráneo.

Callahan, D. y Jennings, B. (2002). Ethics and public health: forging a strong relationship. American Journal of Public Health, 92(2), 169-176.

Calonge, S. y Rodríguez, M. (1998): Consecuencias distributivas y de equidad de las políticas de gasto y financiación de la sanidad. Papeles de Economía Española, 76, 259-272.

Cardozo, C. y Mrad, A. (2013). Investigación científica: una perspectiva ética. En F. Lolas Stepke y J. Freitas Drumond (Eds.), Bioética (pp. 125-138). Santiago de Chile: Mediterráneo.

Cortina, A. (2000). Ética y política: moral cívica para una ciudadanía cosmopolita. Endosa, 12-2, 773-789.

Couceiro, A. (2008). La distribución justa de los recursos en salud. En J. C. Bermejo (Ed.), Salud y justicia (pp. 71-117). Madrid: PPC.

Couceiro, A. (2012). La relación clínica: historia, modelos, instrumentos y retos. En J. P. Beca y C. Astete (Eds.), Bioética clínica (pp. 145-159). Santiago de Chile: Mediterráneo.

Did Núñez, J. (2000). Principios bioéticos en la atención primaria de salud. Revista Cubana de Medicina General Integral, 16(4), 384-389.

Domínguez Márquez, O. y Manrique Nava, C. (2011). Bioética y calidad en la atención de primer contacto y de salud pública. Acta Bioethica, 17(1), 31-36.

Drumond, J. (2010). Ética y calidad en salud. Filotecnia versus filantropía. Acta Bioethica, 16(2), 119-123. 
Escríbar, A. (2004). La actual tendencia a una complementación entre deontología y ética de la responsabilidad. En A. Escríbar, M. Pérez y R. Villarroel (Eds.), Bioética, Fundamentos y dimensión práctica (pp. 104-124). Santiago de Chile: Mediterráneo.

Ferrer, M. (2003). Equidad y justicia en salud: implicaciones para la bioética. Acta Bioethica, 9(1), 113-126.

Garrafa, V. y Porto, D. (2003). Intervention bioethics: a proposal for peripheral countries in a context of power and injustice. Bioethics, 17(5-6), 399-415.

Gracia, D. (2000). Calidad y excelencia en cuidado de la salud. En F. Lolas (Ed.), Bioética y cuidado de la salud. Equidad, calidad, derechos (pp. 35-52). Santiago de Chile: Programa Regional de Bioética OPS/OMS.

Gracia, D. (2001). Bioética clínica. Bogotá: El Búho Ltda.

Gracia, D. (2002). De la bioética clínica a la bioética global: treinta años de evolución. Acta Bioethica, 8(1), 27-39.

Gracia, D. (2012). Fundamentando la ética clínica. En J. P. Beca y C. Astete (Eds.), Bioética clínica (pp. 32-49). Santiago de Chile: Mediterráneo.

Kottow, M. (2012). Bioética crítica en salud pública: ¿aguijón o encrucijada? Revista Chilena de Salud Pública, 16(1), 38-46.

León, F. (2008). De los principios de la bioética clínica a una bioética social para Chile. Revista Médica de Chile, 136, 1078-1082.

León, F., Araya, J. y Nivelo, M. (enero-abril, 2010). Propuesta de comités de bioética en la atención primaria de salud en Chile. Medicina y Humanidades, II(1), 29-40.
León, F. (2010b). Justicia y bioética ante las desigualdades en salud en Latinoamérica. Revista Colombiana de Bioética, 5(1), 85-99.

Lolas, F. (2001). El desafío bioético de la equidad: su relevancia en salud pública. Revista Española de Salud Pública, 75(3), 187-192.

Organización Mundial de la Salud [oms]. (1978). Atención primaria de salud. Conferencia Internacional sobre Atención Primaria de Salud: Alma-Ata. URSS, Ginebra, Suiza.

Organización Panamericana de la Salud [ops]. (2007). Documento de Posición de la Organización Panamericana de Salud/ Organización Mundial de la Salud. Serie: La renovación de la Atención Primaria de Salud en las Américas. Biblioteca Sede OPS; Washington, D.C.

Organización Panamericana de la Salud [ops]. (2008). Sistemas de salud basados en la atención primaria de salud: estrategias para el desarrollo de los equipos de APS. Washington: Biblioteca Sede OPS.

Ortiz, M. y Palavecino, S. (2005). Bioética para una salud pública con responsabilidad social. Acta Bioethica, XI(1), 65-76.

Outomuro, D. (2013a). Derecho a la salud y a la atención en salud. En F. Lolas Stepke y J. G. de Freitas Drumond (Eds.), Bioética (pp. 57-60). Santiago de Chile: Mediterráneo.

Outomuro, D. (2013b). Equidad en salud: cuestiones de la salud pública. En F. Lolas Stepke y J. G. de Freitas Drumond (Eds.), Bioética (pp. 73-80). Santiago de Chile: Mediterráneo.

Oyuela, M. (2010). La importancia de la reconciliación entre Eficiencia y Equi- 
dad, para los sistemas de salud, desde una perspectiva ética. Revista Latinoamericana de Bioética, 10(2), 100-107.

Parenti, F. (2002). Bioética y biopolítica en América Latina. En J. R. Acosta (Ed.), Bioética para la sustentabilidad (pp. 84171). La Habana: Centro Félix Varela.

Rico, V. y Domínguez, O. (2012). Aspectos bioéticos en la toma de decisiones para la calidad de atención en salud. Acta Bioethica, 16(2), 142-147.

Romero, J. (2012). Lo rural y la ruralidad en América Latina: categorías conceptuales en debate. Psicoperspectivas. Individuo y sociedad, 11(1), 8-31.

Rozo, C. (diciembre, 2011). Principios bioéticos y discapacidad: la perspectiva de su inclusión en las políticas públicas. Revista Colombiana de Bioética, 6(2), 26-44.
Rueda, L. (2013). Comités de ética institucional. En F. Lolas Stepke y J. G. de Freitas Drumond (Eds.), Bioética (pp. 37-46). Santiago de Chile: Mediterráneo.

Thompson, A., Robertson, A. y Upshur, R. (2003). Public health ethics: towards a research agenda. Acta Bioethica, 09(2), 157-163.

Yavich, N., Báscolo, E. y Haggerty, J. (2010). Construyendo un marco de evaluación de la atención primaria de la salud para Latinoamérica. Salud Pública de México, 52(1).

Zúñiga, A. (2008). Entre la igualdad y la eficiencia en materia sanitaria. Acta Bioethica, 14(2), 176-184.

Zurro, M., Cano, J. y Gené, J. (2014). Atención primaria. Principios, organización y métodos de medicina en familia (7ma. edición). Madrid: Elsevier. 